\title{
Anti-diabetic activity of red pitaya (Hylocereus polyrhizus) fruit
}

\begin{abstract}
This study investigated the anti-insulin resistant activity of red pitaya (Hylocereus polyrhizus) fruit. Fresh pitaya along with two heat-processed pitaya samples with different proportions of phenolic contents, scavenging activities, and soluble dietary fibers were tested in insulin resistant rats over 6 weeks of treatment in a cause-effect study to investigate the anti-insulin resistant components of red pitaya. The results showed that fresh pitaya significantly $(\mathrm{p}<0.05)$ attenuated the insulin resistance, hypertriglyceridemia and atherosclerotic changes induced by fructose supplement in rats. Thermally-treated pitaya at $95{ }^{\circ} \mathrm{C}$ for $30 \mathrm{~min}$ (containing a low antioxidant content) significantly $(\mathrm{p}<0.05)$ improved hyperinsulinemia. Thermally-treated pitaya at $105{ }^{\circ} \mathrm{C}$ for $60 \mathrm{~min}$ (containing a low antioxidant and soluble dietary fiber content) fed to rats had no significant effect on insulin resistance, dyslipidemia and atherogenesis. Based on this study, the anti-insulin resistant effect of red pitaya can be attributed to its antioxidant and soluble dietary fiber contents.
\end{abstract}

Keyword: Anti-insulin resistant; Hylocereus polyrhizus; Red pitaya 